\title{
Formation of surface color centers at differently coordinated sites: $\mathrm{MgO} / \mathrm{Ag}(1,1,19)$
}

\author{
J. Kramer, C. Tegenkamp, and H. Pfnür* \\ Institut für Festkörperphysik, Universität Hannover, Appelstraße 2, D-30167 Hannover, Germany
}

(Received 14 February 2003; published 5 June 2003)

\begin{abstract}
In order to find criteria for discrimination between surface color centers at terraces and steps, and bulk color centers, the characteristic losses of these centers have been investigated by electron energy loss spectroscopy on an epitaxial $\mathrm{MgO}$ film grown on a $\mathrm{Ag}(1,1,19)$ surface. This film contains a significantly higher step density compared with a film grown on $\mathrm{Ag}(100)$. The generation of four distinct losses at 2.0, 2.4, 2.8, and $3.4 \mathrm{eV}$ and a broad loss centered at $5.5 \mathrm{eV}$ have been observed that are induced by electron bombardment of the $\mathrm{MgO}$ surface. The latter loss is attributed to bulk color centers $\left(F_{b}\right.$ centers). By comparing the measured loss energies with experiments performed on $\mathrm{MgO} / \mathrm{Ag}(100)$ and with theoretical literature data, the observed losses at 2.0 and $2.8 \mathrm{eV}$ can be consistently assigned to transitions of color centers located on step sites, whereas those at 2.4 and $3.4 \mathrm{eV}$ are attributed to terrace sites. The kinetics of color center formation during electron bombardment, as well as the annihilation of $\mathrm{F}$ centers by simple exposure to $\mathrm{O}_{2}$ gas at room temperature, was determined and compared for differently coordinated color centers. While all surface color centers follow the same kinetics, we show that the $F_{b}$ centers can only decay as long as the existence of surface color centers provides active sites for dissociating oxygen molecules. This corroborates the assignment of $F_{b}$ centers.
\end{abstract}

DOI: 10.1103/PhysRevB.67.235401

PACS number(s): 77.84.Bw, 68.35.Dv, 61.72.Ji

\section{INTRODUCTION}

The properties of insulating material with wide band gaps, especially at their surfaces and interfaces, is to a large extent governed by the properties and concentrations of defects. The reason is that the perfect surfaces of such materials, e.g., of most metal oxides, are chemically highly inert, ${ }^{1}$ since they form no surface states, so that at the surface the band gap is only slightly reduced compared with the bulk. Therefore, detailed investigations of the physical properties of defects at insulator surfaces are necessary in order to come to a deeper understanding of their role in the wide range of applications, where these materials are used. It spans from catalysis, ${ }^{2,3}$ surface passivation to the fabrication of electronic devices. ${ }^{4,5}$

The growth of ultrathin epitaxial oxide films has greatly improved the possibilities for their detailed investigations. Several studies have been carried out for magnesium oxide. $^{6-9}$ They form the basis for well defined studies of defect properties, so that a direct comparison with the wealth of theoretical results will be possible. In several theoretical papers ${ }^{10-13}$ the properties of surface color centers $F_{s}$ on $\mathrm{MgO}$ have been investigated. Both the electronic structure of $F$ and $V$ centers (magnesium vacancy) and the optical properties have been calculated by means of cluster models and $a b$ initio wave functions and by first principles calculations. Also the formation of $\mathrm{Mg}-\mathrm{O}$ divacancies has been studied. ${ }^{14}$ The energy gain by the formation of a divacancy out of two isolated vacancies is very high. Divacancies generated in the bulk tend to migrate to the surface. This result is in agreement with the stability of oxygen vacancies on subsurface, terrace, and low coordinated surface sites. ${ }^{15} \mathrm{Ab}$ initio HartreeFock cluster calculations suggest that $F$ centers at lowcoordinated sites are more stable than at high-coordinated sites.

The detailed theoretical information contrasts with few published experimental results that deal with the properties and the formation of $F_{s}$ centers on $\mathrm{MgO} .^{9,16-18}$ These have been partly inconclusive and even contradictory. Early work $^{18}$ found a broad electronic loss between 1 and $3.5 \mathrm{eV}$ induced by electron irradiation of a $\mathrm{MgO}$ surface, which may consist of several unresolved loss peaks. Further experiments examined both the formation of color centers during high temperature annealing of $\mathrm{MgO}$ films on $\mathrm{Mo}(100),{ }^{9}$ and the defect generation by $\mathrm{Ar}^{+}$bombardment and by evaporation of additional $\mathrm{Mg}$ onto the $\mathrm{MgO}$ surface. ${ }^{17}$ The observed loss peaks in both experiments are not the same, but no obvious reason for this discrepancy could be given.

This motivated us to carry out additional experiments with electron energy loss spectroscopy (EELS) to resolve this unsatisfying situation at least partly. First we examined the formation of $F_{s}$ centers induced by electron bombardment of $\mathrm{MgO}$ films grown on an $\mathrm{Ag}(100)$ surface. ${ }^{16}$ The generation of five distinct losses has been observed. By comparing our experimental data with theoretical results, ${ }^{10}$ an assignment of the observed transition energies to $F_{s}$ centers coordinated at terrace and step sites was possible. To verify these assignments, we now increased the step density of the $\mathrm{MgO}$ films by using a vicinal silver substrate. These results are presented in this study.

The paper is organized as follows. After a short experimental section, we discuss the origin of the electronic losses found, when an $\mathrm{MgO}$ film with a high step density, named $\mathrm{MgO}_{A g, v i c}$ in the following, is bombarded with electrons by comparing these results with the measurements performed on an $\mathrm{MgO}$ film grown on $\mathrm{Ag}(100)$. Section III is devoted to the kinetics of generation and annihilation of surface color centers at differently coordinated sites, tested also on the $\mathrm{MgO}_{A g, v i c}$ surface, before we summarize our results.

\section{EXPERIMENT}

The experiments have been performed in a ultrahigh vacuum chamber operated at a base pressure of 1 $\times 10^{-8} \mathrm{~Pa}$. It was equipped with a high- resolution low en- 
ergy electron diffraction (LEED) to control the morphology of the silver sample and of the $\mathrm{MgO}$ film. The electronic structure was studied using $\mathrm{He}$ I and $\mathrm{He}$ II radiation for ultraviolet photoemission spectroscopy (UPS), and an Al target for x-ray induced photoemission (XPS). For EELS measurements, electrons with a primary energy of $50 \mathrm{eV}$ have been focussed onto the sample at an angle of $60^{\circ}$ with respect to the surface normal, and the specularly reflected electrons with their characteristic losses have been measured. Thus we are sensitive primarily to dipolar losses. The photoelectrons and the backscattered electrons from EELS have been detected by a $150^{\circ}$ spherical analyzer $(r=100 \mathrm{~mm})$.

For this study a $\operatorname{Ag}(1,1,19)$ surface was used. The sample was mounted on a transferable sample holder. This holder also included a thermocouple ( $\mathrm{Ni} / \mathrm{Ni}-\mathrm{Cr})$ connected to the sample holder near the sample surface, and a filament located behind the sample for heating it by radiation. All measurements have been performed at room temperature. The $\operatorname{Ag}(1,1,19)$ surface is vicinal to $(100)$ with steps in the [ $\overline{1} 10]$ direction. The mean distance between steps on the ideal surface is about $28.3 \AA$.

Surface cleaning in vacuum was achieved by sputtering and annealing cycles $\left(\mathrm{Ar}^{+}\right.$sputtering at room temperature for $20 \mathrm{~min}$ at $2 \mathrm{keV}$, annealing up to $700 \mathrm{~K}$ ). In order to achieve a clear spot splitting in LEED, a slow controlled cooling of the sample to room temperature was necessary. Surface cleanness was controlled by XPS.

Thin $\mathrm{MgO}$ films of 8-ML thickness were grown on the $\mathrm{Ag}(1,1,19)$ substrate at room temperature by evaporating $\mathrm{Mg}$ in a $\mathrm{O}_{2}$ partial pressure of $2 \times 10^{-5} \mathrm{~Pa}$ (Refs. 6 and 17) at an evaporation rate of $R=0.3 \mathrm{ML} / \mathrm{min}$. After preparation, the films have been annealed up to $700 \mathrm{~K}$ for at least half an hour. The thickness of the films used in these experiments was estimated by using a quartz microbalance and the calibration carried out previously. ${ }^{17}$

In order to create color centers, a thoriated tungsten filament was placed in front of the sample a few millimeters away from the surface. The surface was at temperatures close to room temperature. The sample was set to positive voltage with respect to the filament, which was connected to ground, and the emitted electron current was measured at the sample. The electron exposure was calculated from this current assuming a homogeneous distribution of current density across sample surface, and is given as the number of electrons per surface unit cell, which is equal to the number of electrons per surface oxygen ion.

\section{RESULTS AND DISCUSSION}

The $\mathrm{MgO}$ films examined in this study have been grown on an $\operatorname{Ag}(1,1,19)$ substrate. The morphology of $\mathrm{MgO}$ films grown on this substrate as a function of coverage and growth temperature has been studied in detail by spot profile analysis in low energy electron diffraction. ${ }^{8}$ Starting with a thickness of $5 \mathrm{ML}$, the $\mathrm{MgO}$ films consist of mosaics with characteristic angles of inclination between $0.9^{\circ}$ and $1.5^{\circ}$ and of nontilted $\mathrm{MgO}$ crystallites. Both cover about $50 \%$ of the surface. The detailed spot profile analysis of the non-inclined parts of the $\mathrm{MgO}$ layers revealed that the steps are mostly one atom high. Assuming that the step density on the mosaics and on the non-inclined crystallites is approximately the same, the step density on $\mathrm{MgO}_{A g, v i c}$ is estimated to be at least $30-50 \%$ higher than on $\mathrm{MgO}_{A g, \text { flat }}$.

As mentioned, the closer identification of characteristic losses due to color centers is the main motivation of this investigation. Therefore, here we compare the results obtained on an $\mathrm{MgO}$ surface with high step density, generated after growth of the film on the vicinal $\operatorname{Ag}(1,1,19)$ surface $\left(\mathrm{MgO}_{A g, v i c}\right)$, with those obtained on $\mathrm{MgO}_{\text {Ag, flat }}$.

After preparation, the stoichiometry of each $\mathrm{MgO}$ film was investigated by XPS. No splitting of the $\mathrm{O} 1 \mathrm{~s}$ and of the $\operatorname{Mg}(K L L)$ peaks has been observed that would be characteristic for metallic or non-stoichiometric components. ${ }^{6}$ Therefore, we conclude that only stoichiometric $\mathrm{MgO}$ has been formed during our preparation.

\section{A. Formation of color centers}

Recently we have examined the formation of color centers on an $\mathrm{MgO}$ film on $\operatorname{Ag}(100)$ generated by electron bombardment. ${ }^{16}$ The formation of five distinct loss features at 1.0, 1.3, 2.4, 2.8, and $3.4 \mathrm{eV}$ was observed. The comparison with theoretical calculations performed by Sousa et al. ${ }^{10}$ allowed a surprisingly close correlation of the calculated excitation energies (after a rigid shift by $1 \mathrm{eV}$ ) of color centers at various locations (terrace, step, and kink) with the measured loss energies. The loss energies 2.4 and $3.4 \mathrm{eV}$ coincide closely with the calculated transitions of color centers located at a terrace site, where the $1 s \rightarrow 2 p_{z}$ transition $\left(T_{z}\right)$ should be responsible for the $2.4-\mathrm{eV}$ loss and $1 s \rightarrow 2_{x, y}$, $\left(T_{x, y}\right)$ for the other one. The measured loss energy of $2.8 \mathrm{eV}$ can be correlated with the electronic transition $1 s \rightarrow 2_{x, y}$, $\left(S_{x, y}\right)$ of a low-coordinated color center at a step site. The calculated excitation energy for the $1 s \rightarrow 2 p_{z},\left(S_{z}\right)$ transition of a color center at a step site of $1.92 \mathrm{eV}$ did not correspond to a clear peak in this energy range of the experimental data. Although there was significant intensity at this loss energy a peak was not resolved, most likely because of the more intense features at other close loss energies. If the assignment is correct, the peaks correlated with color centers located at steps like the " $S_{z}$ " peak should get more weight on a surface with higher step density.

Finally, the loss peaks at 1.0 and $1.3 \mathrm{eV}$, not found in the calculations, which have been restricted to single color centers, ${ }^{10}$ may be due to electronic transitions of $M$ centers. In Fig. 1, we compare the results obtained on $\mathrm{MgO}$ films grown on $\operatorname{Ag}(100)$ (top) with those on $\operatorname{Ag}(1,1,19)$ (bottom). The EEL spectra of the freshly prepared $\mathrm{MgO}$ films without color centers are shown for reference as dashed lines in both parts of the figure. Both spectra exhibit a nearly perfect band gap. Within the band gap, only the $\mathrm{MgO}-\mathrm{Ag}$ interface plasmon excitation is seen at a loss energy of $3.4 \mathrm{eV}$. The only main difference between the two spectra is that the loss at 6.2 $\mathrm{eV}$, corresponding to surface related excitonic excitation, ${ }^{19}$ is much less pronounced on the stepped surface. When normalized to the elastic peak intensity (not shown here), this reduction appears even more drastic, since it amounts to a factor of 2. This indicates that this exciton is not strongly localized and is distorted by the higher step density of the 

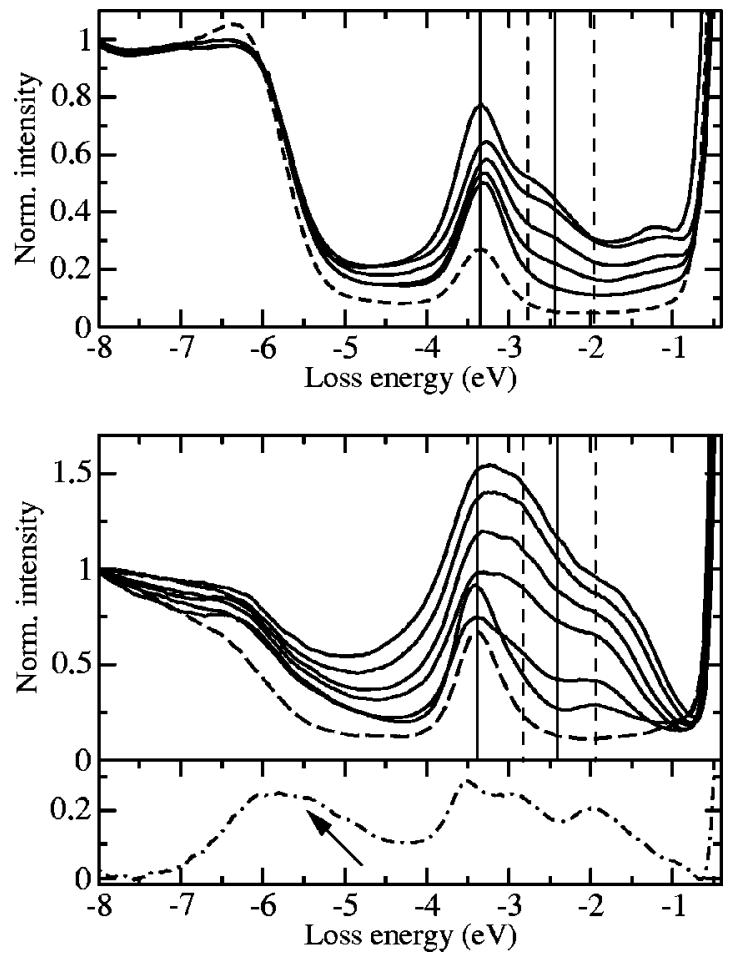

FIG. 1. EEL spectra of color centers generated by electron bombardment at $150-\mathrm{eV}$ incident energy as a function of increasing electron dose (from bottom to top) on $\mathrm{MgO}$ films grown on $\mathrm{Ag}(100)$ and on $\operatorname{Ag}(1,1,19)$. The dashed curves are measured immediately after growth. Upper panel: 10-ML $\mathrm{MgO} / \mathrm{Ag}(100)$. 180, 900, 1440, 2880, and 3780 electrons per surface oxygen ion. Lower panel: 8-ML MgO/Ag(1,1,19). 70, 140, 200, 380, 830, 1280, 2180, and 3980 electrons per surface oxygen ion. The tentative assignments of transitions are marked by vertical solid lines for color centers located on a terrace site and by dashed lines for those located at steps. The lowest curve (dash-dotted) marks the difference spectrum between the first two curves shown. The intensity most likely due to bulk $\left(F_{b}\right)$ centers is marked with an arrow.

$\mathrm{MgO}$ film grown on the vicinal $\mathrm{Ag}$ surface. In addition, the lifetime of the excitonic excitation may be reduced due to the increased step density. The same behavior was observed by Tegenkamp et al. ${ }^{20}$ for $\mathrm{NaCl}$ grown on vicinal Ge with various step densities.

The main part of Fig. 1 shows the build-up of characteristic losses due to the formation of $F_{s}$ centers on $\mathrm{MgO}_{A g, \text { flat }}$ (top) and on $\mathrm{MgO}_{A g, v i c}$ (bottom) as a function of electron exposure up to a total dose of 4000 electrons per surface oxygen ion during bombardment of the film with electrons of an incident energy of $150 \mathrm{eV}$. The conditions for the generation of color centers are the same in both cases. The position of peaks assigned to $F_{s}$ centers on terrace sites and step sites are marked by perpendicular solid and dashed lines.

The formation of several characteristic losses can be observed in both parts of the figure. For the $\mathrm{MgO}_{A g}$,flat film, the already known losses are generated. Here the pairs of peaks at 2.4 and 2.8 and at 1.0 and $1.3 \mathrm{eV}$ are not clearly resolved but have been resolved for other bombardment energies. On the $\mathrm{MgO}_{A g, v i c}$ surface the loss peaks appear at the same energies, but with a different weight. This difference in relative weight is especially pronounced for the intensity at $2.0 \mathrm{eV}$, which shows up as an additional peak. By comparison with the calculations of Sousa et al. ${ }^{10}$ this peak has been assigned to the $S_{z}$ transition at step edges. Since the $\mathrm{MgO}_{A g, v i c}$ film is expected to have a higher step density than the $\mathrm{MgO}_{A g, \text { flat }}$ films, a higher probability for the generation of color centers at step edges on the former is consistent with the assignment of the $S_{z}$ transition. The dominance of this peak makes it impossible to resolve the loss pair at 1.0 and $1.3 \mathrm{eV}$ with the given experimental resolution, which can be clearly seen on the flat surface. The different weights of color center formation at step edges and on flat terraces for the $\mathrm{MgO}_{A g \text {, flat }}$ and the $\mathrm{MgO}_{A g \text {,vic }}$ films can be quantified by taking the ratios $S_{x, y} / T_{x, y}$ and $S_{z} / T_{z}$. An increase of the $S / T$ ratios by roughly $40 \%$ is observed for the color centers created on $\mathrm{MgO}_{A g, v i c}$. Assuming, that the density of $F_{s}$ centers on terrace sites at a given electron dose is the same for both films, an increase of the step density by this amount follows on $\mathrm{MgO}_{A g, v i c}$ compared with $\mathrm{MgO}_{A g, f l a t}$. This increased step density corresponds closely to estimates from data in Ref. 8. For the part of the $\mathrm{MgO}$ film not forming mosaics, we found an average terrace length of $37 \AA$ in the direction parallel to the step edges. As a first approximation this value is representative for $\mathrm{MgO}_{A g, \text { flat }}$. On the $\mathrm{MgO}_{A g, v i c}$ there are additional steps due to the vicinality. There we obtained a value of $27.9 \AA$ for the average terrace length, which can also be taken as a lower limit for the step separation for the $\mathrm{MgO}$ film. Although the roughness parameters were derived for a 5-ML-thick MgO film, they should be more or less the same for slightly thicker $\mathrm{MgO}$ films used in this study. As a consequence the step density in the [110] direction is increased at least $30 \%$. This value is in agreement with the increased S/T-ratio on $\mathrm{MgO}_{A g, v i c}$ compared to $\mathrm{MgO}_{A g, \text { flat }}$. The increase of the $S / T$ ratio, resulting in a clear loss peak at $2.0 \mathrm{eV}$ on $\mathrm{MgO}_{A g, v i c}$, corroborates the assignment of the losses already made, ${ }^{16}$ i.e., to $F_{s}$ centers on terrace and on step sites.

In Fig. 1 a further difference between the two bombarded $\mathrm{MgO}$ surfaces was observed. While there are only small changes between the fresh and the bombarded surfaces close to the left margin of the band gap on $\mathrm{MgO}_{A g, \text { flat }}$, an increase of the loss intensity was observed on $\mathrm{MgO}_{A g, v i c}$. In addition, the intensity of the plasmon decreases strongly during electron bombardment of $\mathrm{MgO}_{A g, v i c}$, an effect not observed on $\mathrm{MgO}_{\text {Ag, flat }}$. In fact, an additional loss peak has been created with a loss energy around $5.5 \mathrm{eV}$, which becomes evident when subtracting loss spectra of the bombarded films form a fresh one (see the bottom curve of Fig. 1). This broad loss peak may be attributed to the existence of bulk color centers ( $F_{b}$ centers), but may also contain shifts or broadening of the $\mathrm{MgO}$ excitonic excitation during bombardment (also see below). Chen and co-workers ${ }^{21,22}$ found an absorption band in the vicinity of $5.0 \mathrm{eV}$ for $F_{b}$ centers. Wu et al. ${ }^{9}$ examined the thermal generation of defects in ultrathin $\mathrm{MgO}$ films with high- resolution EELS. They found a loss peak at $5.3 \mathrm{eV}$, which has been associated with $F_{b}$ centers. These findings make it likely that the broad loss peak seen in our experiments on $\mathrm{MgO}_{A g, v i c}$ contain contributions from losses due 
to $F_{b}$ centers. Also the loss intensity of the $\mathrm{MgO}-\mathrm{Ag}$ interface plasmon at $3.4 \mathrm{eV}$ is affected by electron bombardment. These experiments show that the electron bombardment also induces disorder near the interface, which results in a smaller plasmon intensity.

Compared to $\mathrm{MgO}_{A g, \text { flat }}$, the observed intensity related with $F_{b}$ centers is much larger on $\mathrm{MgO}_{A g, v i c}$. Since the primary step for color center generation involves core level excitations and their Auger decay, ${ }^{16}$ this process should be independent of surface roughness or step density. Therefore, only the probability for $F_{b}$ centers to survive may be different on rougher $\mathrm{MgO}$ surface. Indeed the diffusivity of oxygen atoms to the surface necessary to stabilize an $F_{b}$ center may be higher on rough surfaces for color centers created close to the surface. Since $F_{b}$ centers are completely embedded in a dielectric medium, their cross section for detection is always smaller than for $F_{s}$ centers, depending on the dielectric function of the medium. This effect may be reduced due to a reduction of the effective dielectric constant of the $\mathrm{MgO}$ film ${ }^{23}$ because of additional roughness of the film on a length scale much larger than the average terrace width. However, this length scale cannot be studied in LEED experiments, so that its influence cannot be quantified.

The spectra shown in Fig. 1 are normalized to a loss energy of $8 \mathrm{eV}$. The normalization to a loss energy of $8 \mathrm{eV}$ was chosen because no characteristic losses and a smooth behavior of the inelastic background are observed in this energy range. Although the spectra of the flat and the vicinal surface are normalized to the same point, the absolute intensities cannot be compared between both surfaces. As mentioned above, the intensity of the excitonic excitation is reduced by a factor of 2 for the vicinal surface. Therefore, and contrary to their appearance, the observed densities for color centers on both surfaces are very close to each other.

\section{B. Kinetics of color center creation}

In the following we describe a study of the defect generation on the $\mathrm{MgO}_{A g, v i c}$ surface. In Fig. 2 the peak height, which is characteristic of the defect concentration $n_{s}$, measured by intensities of the $S_{z}, T_{z}$, and $S_{x, y}$ transitions, are shown versus the electron exposure for the $\mathrm{MgO}_{A g, v i c}$ film. In addition, the formation of the bulk color center is shown (will be discussed later). It was not possible to analyze the $T_{x, y}$ transition quantitatively, because of the unknown variation of the loss intensity of the interface plasmon as a function of electron dose.

Similar to the behavior found on $\mathrm{MgO}_{A g, \text { flat }}$ films,${ }^{16}$ the generation of defects on different coordinated sites as a function of electron dose $D$ can again be described by a first order rate equation, as shown in Fig. 2 by the fits to the data:

$$
\frac{d n_{s}}{d D}=a\left(1-n_{s}\right)-b n_{s} \Rightarrow n_{s}(D)=\frac{a}{a+b}\left(1-e^{(a+b) D}\right) .
$$

The first term describes the defect generation and the second part the possible annihilation. The constant $a$ is a measure for the cross section of color center generation. ${ }^{16}$ The saturation concentration is determined by $a /(a+b)$. As is obvious from Fig. 2, both $S$ and $T$ transitions follow the same first

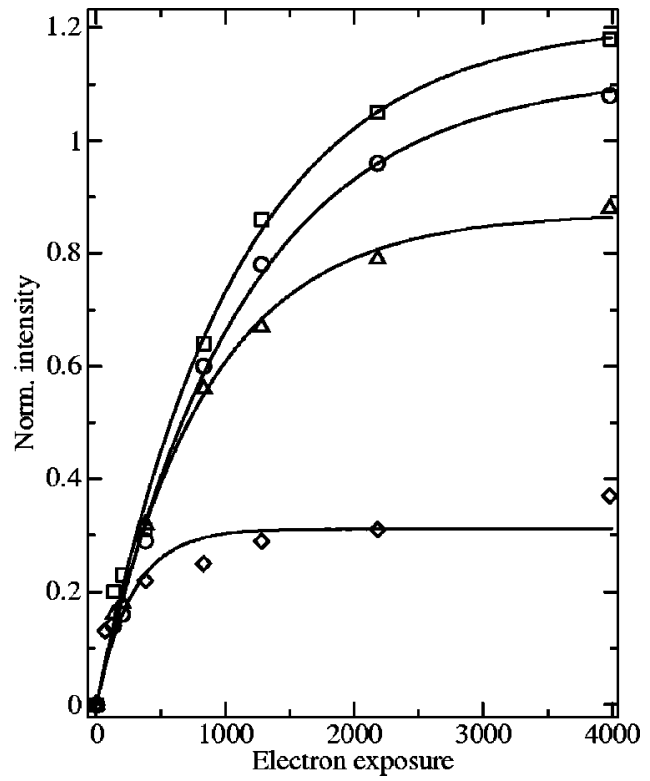

FIG. 2. Intensity evolution of the loss peaks of the stepped surface as a function of electron exposure at a bombardment energy of $150 \mathrm{eV}$ for the following losses: $(\triangle) 2.0 \mathrm{eV}, S_{z}$, (○) $2.4 \mathrm{eV}, T_{z}$, ( $\square) 2.8 \mathrm{eV}, S_{x, y}$, $(\diamond) 5.6 \mathrm{eV}, F_{b}$ center. The solid lines show the fits to the data $\propto 1-e^{(a+b) D}$. Electron exposure is in units of electrons per surface oxygen atom.

order kinetics. Furthermore, similar time constants and saturation concentrations for the $F_{s}$ centers have been found compared with the $\mathrm{MgO}_{A g, f l a t}$ films. This means that the general mechanisms for color center formation and destruction are the same on both types of $\mathrm{MgO}$ films, as expected.

We note that the generation rate in the initial stage differs from that at the higher doses shown in Fig. 1. At the beginning, the $2.0-\mathrm{eV}$ peak increases much faster than the $2.4-\mathrm{eV}$ peak. After a dose of about 200 electrons per oxygen ion the situation turns. This behavior indicates that the generation of color centers on step sites is initially preferred. On the other hand, the destruction of this defect is more effective in a later stage.

The formation of color centers on $\mathrm{MgO}$ occurs via a Knotek-Feibelman mechanism. ${ }^{16,24,25}$ This is a multielectron Auger decay process, which begins with the excitation of core levels of either $\mathrm{Mg}$ or $\mathrm{O}$. The holes can decay in an interatomic process, which results in the formation of a neutral oxygen atom or a positive oxygen ion. The oxygen atom might still be slightly bound to the surface, but the positive ion is no longer stable since the Madelung term becomes repulsive. Once the oxygen is desorbed an $F$ center is formed. This mechanism is not expected to be changed for color centers formed at differently coordinated sites. The only difference is the lower coordination at step edges compared to terrace sites. As a consequence, it might be easier for the oxygen atom/ion to desorb from the step than from the terrace. As a consequence the cross section for $F_{s}$ center generation on step sites is larger initially, which is in good agreement with the experiment.

As can be seen in Fig. 2, also the formation of $F_{b}$ centers follows the same first order kinetics than the $F_{s}$ centers. We 


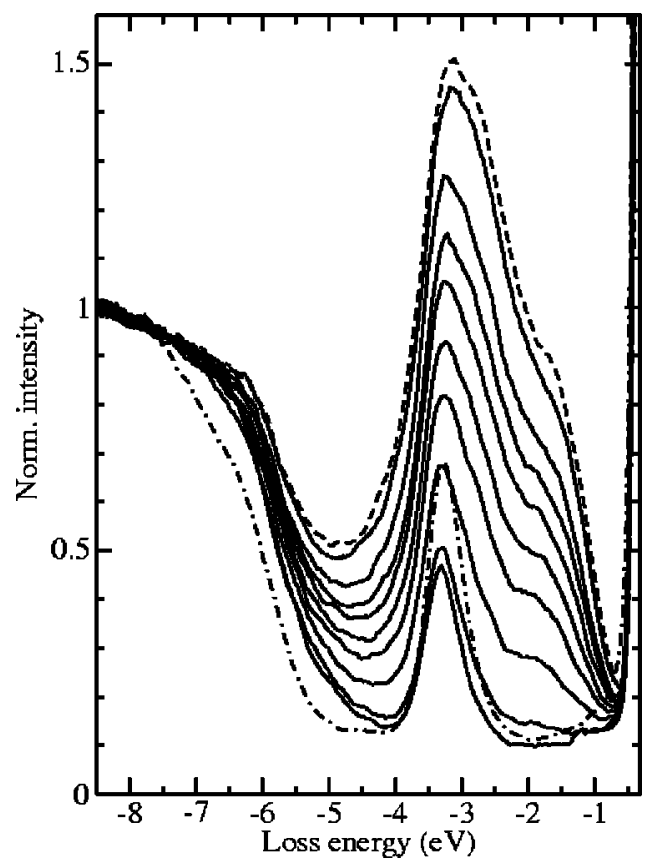

FIG. 3. EEL spectra of the vicinal $\mathrm{MgO}$ surface as in Fig. 1 before (dash-dotted curve, bottom) and after electron bombardment with an incident energy of $150 \mathrm{eV}$ (dashed curve, top). From top to bottom (solid curves): measurement after increasing exposure with oxygen at room temperature $(0.04,0.11,0.16,0.21,0.27,0.34$, $0.48,1.0$, and $2.0 \mathrm{~L})$.

therefore conclude that the coordination of the color centers does not influence the mechanism of defect formation, only the kinetic parameters are slightly dependent on the coordination. The reverse process, defect annihilation, however, seems to vary for differently coordinated color centers. This will be described in the next section.

\section{Annihilation of color centers by oxygen exposure}

Annihilation of color centers is possible by several mechanism. An $F$ center disappears, if an $\mathrm{Mg}$ atom located next to an oxygen vacancy is removed, either by thermal desorption or by excitation during electron bombardment. This mechanism might become more likely for $\mathrm{Mg}$ atoms next to low-coordinated $F_{s}$ centers, because of the reduced number of bonds. This would reduce the saturation concentration of color centers located at step edges compared to color centers at terrace sites, and can explain the weak increase of the 2.0-eV peak at higher electron doses (discussed in the previous subsection). Alternatively, an $F_{s}$ center can be filled by adsorbing atoms from the residual gas.

As described in Ref. 16, the destruction of color centers at the surface follows an exponential decay, if the surface is exposed to gases containing oxygen. This means that the molecules from the residual gas are adsorbed on the surface and diffuse randomly for a finite time and samples a certain area of the surface. If they find an oxygen vacancy during their life time, they react. Since diffusion is expected to happen with different diffusion constants along step edges and across terraces, it is conceivable that effective time constants

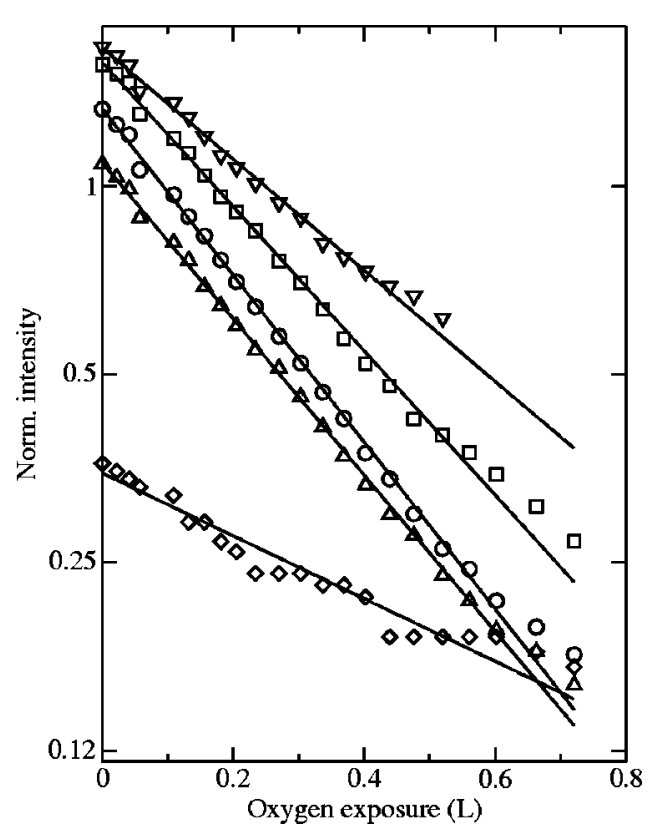

FIG. 4. Semilog plot of the loss intensities as a function of oxygen exposure at different loss energies: $(\triangle) 2.0 \mathrm{eV}, S_{z}$, (O) 2.4 $\mathrm{eV}, T_{z},(\square) 2.8 \mathrm{eV}, S_{x, y},(\nabla) 3.4 \mathrm{eV}, T_{x, y}$, and $(\diamond) 5.6 \mathrm{eV}, F_{b}$ centers. The fits (lines) have been carried out with simple exponential functions.

for extinction differ between color centers at step edges and at terraces. This would change the constant of decay and as a consequence, also the saturation constant.

These inherent questions have been answered by an adsorption experiment with oxygen. We exposed the $\mathrm{MgO}$ surface to oxygen after bombardment with electrons. When the oxygen pressure is much higher than the base pressure of the chamber, the adsorption of oxygen will be the main decay channel so that all other decay channels can be neglected.

An EEL spectra of the initial surface can be seen at the bottom of Fig. 3 (dash-dotted curve), whereas the topmost curve (dashed curve) shows the generated loss bands after electron bombardment at saturation. From Fig. 3 it is evident that during oxygen exposure all intensities of the $F_{s}$ peaks are reduced simultaneously.

The dose was increased in unequal steps up to $3.0 \mathrm{~L}$. Already an exposure of $2.0 \mathrm{~L}$ was sufficient to extinguish all losses associated with $F_{s}$ centers (see Fig. 3). In contrast, the loss intensity in the region between 5 and $6 \mathrm{eV}$ is not fully reduced to the original level before electron bomardment, i.e. the loss spectrum of the undisturbed surface was not restored by an oxygen dose up to 3.0-L oxygen. This means, that only a part of the $F_{b}$ centers have been annihilated. The effective time constants of decay as a function of oxygen exposure have been determined from a semilog plot of the five observed intensities, shown in Fig. 4.

The fits (lines) have been carried out with simple exponential functions. This plot shows that within some scatter the transitions assigned to $F_{s}$ centers on differently coordinated sites and the $F_{b}$ centers both follow an exponential decay. It is interesting to note that the $S_{z}$ and $S_{x, y}$ peaks decay nearly with the same time constant as the $T_{z}$ and $T_{x, y}$ peaks, whereas the constant for the $F_{b}$ centers is much 


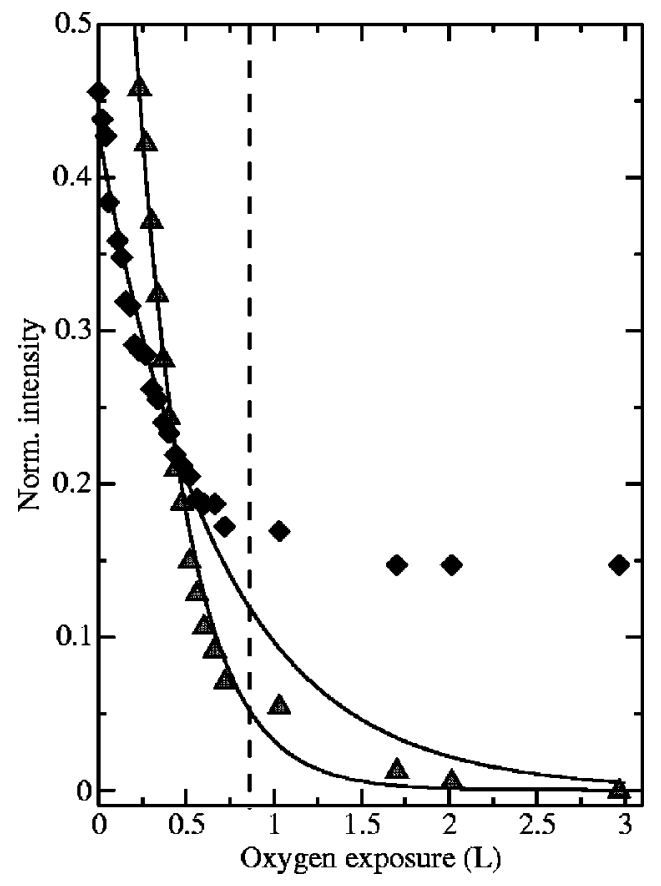

FIG. 5. Cutout of Fig. 4 for $(\triangle) 2.0 \mathrm{eV}, S_{z}$ and $(\diamond) 5.6 \mathrm{eV}, F_{b}$ centers. For higher oxygen exposures than $0.8 \mathrm{~L}$ (dashed line) the loss intensity induced by $F_{b}$ centers does not show an exponential decay anymore.

smaller (this will be discussed below). Deviations from the exponential decay at high-oxygen exposures are most likely due to errors in background subtraction. This shows that the anisotropy of the $\mathrm{MgO}$ surface due to steps is of no importance for the decay of color centers during oxygen exposure at temperatures close to room temperature, leading to equal decay constants for F centers at steps and at terraces.

As mentioned above, the time constant for the decay of $F_{b}$ centers is much smaller than for the $F_{s}$ centers. Furthermore, there is a significant deviation from a simple exponential decay for higher oxygen exposures.

In Fig. 5 it is shown that the intensity associated with $F_{b}$ centers levels off for oxygen exposures higher than $0.8 \mathrm{~L}$. At this exposure nearly all $F_{s}$ centers are annihilated, as demonstrated for the $S_{z}$ transition. This suggests a close relationship between the existence of color centers at the $\mathrm{MgO}$ surface and the capability of annihilation of bulk $F$ centers, which can easily be understood by the following model. In order to delete an $F_{b}$ center, a single oxygen atom must be created and diffuse into deeper layers. Since oxygen is adsorbed as a molecule, it must find a site to dissociate. The only reactive defects on the surface capable of dissociating oxygen molecules are the $F_{s}$ centers present on the surface. As a consequence, an annihilation of bulk color centers always starts with the filling of an $F_{s}$ center, which creates an extra oxygen atom. There is a certain probability for this atom to penetrate the surface and reach a bulk $F$ center by diffusion, but this process becomes irrelevant as soon as all $F_{s}$ centers are filled and no free oxygen atoms are created. This shows, that the decay of color centers in deeper layers must be slower than the decay of $F_{s}$ centers. It stops at temperatures close to room temperature, where thermal generation of oxygen atoms is negligible, when all $F_{s}$ centers are filled, as observed. This leaves most likely a certain fraction of unfilled color centers in deeper layers after filling all surface color centers, as observed in the experiment as remaining intensity between 5- and 6-eV loss energy.

This short discussion makes clear, that oxygen molecules are dissociated at $F_{s}$ centers. The annihilation of $F_{s}$ centers due to oxygen exposure was explained in Refs. 26 and 27 as an electron transfer from the $F_{s}$ center to the $\mathrm{O}_{2}$ and the formation of a $\mathrm{O}_{2}^{-}$, in agreement with theoretical calculations of Pacchioni and co-workers. ${ }^{28,29}$ These authors found that $\mathrm{O}_{2}$ acts as an electron acceptor and destroys the $F_{s}$ centers by the formation of superoxide anion at the expense of a high activation barrier for the ionization of the $\mathrm{O}_{2}$. Our experiments also make it clear that there are no other defect than the $F_{s}$ centers on the surface that are able to dissociate an oxygen molecule. Otherwise the annihilation of $F_{b}$ centers would not stop after all $F_{s}$ centers have been deleted. As a consequence, terraces and steps of the $\mathrm{MgO}$ surface do not react with $\mathrm{O}_{2}$.

\section{SUMMARY AND CONCLUSION}

The higher step density of the $\mathrm{MgO}$ films grown on $\operatorname{Ag}(1,1,19)$ that have been used in this study, allowed the verification of the assignment of the loss peaks in EELS (Ref. 16) induced by surface color centers that have been generated by electron bombardment of the $\mathrm{MgO}$ films. This means that the losses at energies of 2.4 and $3.4 \mathrm{eV}$ are caused by $F_{s}$ centers on terrace sites, whereas the peaks at 2.0 and $2.8 \mathrm{eV}$ are attributed to color centers at step sites. In addition, the formation of $F_{b}$ centers was observed with a broad characteristic loss around $5.5 \mathrm{eV}$.

The generation of all observed losses follows the same first order kinetics. As a consequence, the general mechanism of color center formation and annihilation is not influenced by the coordination of the $F$ center, only the kinetic parameters are slightly changed.

In contrast to the $F_{b}$ centers, the $F_{s}$ centers are completely erased by oxygen exposure, following an exponential decay with similar time constants for differently coordinated color centers. Therefore, the anisotropy of the $\mathrm{MgO}$ surface due to steps is of no importance for the decay of $F_{s}$ centers during oxygen exposure at room temperature.

The annihilation of the $F_{b}$ centers, on the other hand, ends when all $F_{s}$ centers are destroyed. From this fact we conclude, that it is necessary for $F_{b}$ centers to be annihilated that $\mathrm{O}_{2}$ molecules are dissociated at $F_{s}$ centers. These data may be used as basis for detailed studies of the reactivities of color centers or other species. 
*Electronic address: pfnuer@fkp.uni-hannover.de

${ }^{1}$ V. E. Henrich and P. A. Cox, The Surface Science of Metal Oxides (Cambridge University Press, Cambridge, 1994).

${ }^{2}$ C. $\mathrm{Xu}$ and D. W. Goodman, in Handbook of Heterogeneous Catalysis, edited by G. Ertl, H. Knözinger, and J. Weitkamp (Verlag Chemie, Weinheim, 1997).

${ }^{3}$ S. Abbet, U. Heiz, A.M. Ferrari, L. Giordano, C. Di Valentin, and G. Pacchioni, Thin Solid Films 400, 37 (2001).

${ }^{4}$ C.D. Fung, P.W. Cheung, and W.H. Ko, IEEE Trans. Electron Devices ED-33, 8 (1986).

${ }^{5}$ F. Assaderaghi, S. Parke, P.K. Ko, and H. Chenming, IEEE Symp. Low Power Electr. 104, 58 (1994).

${ }^{6}$ J. Wollschläger, J. Viernow, C. Tegenkamp, D. Erdös, K.-M. Schröder, and H. Pfnür, Appl. Surf. Sci. 142, 129 (1999).

${ }^{7}$ J. Wollschläger, D. Erdös, H. Goldbach, R. Höpken, and K.-M. Schröder, Thin Solid Films 400, 1 (2001).

${ }^{8}$ J. Kramer, C. Tegenkamp, W. Ernst, and H. Pfnür, Surf. Sci. (to be published).

${ }^{9}$ M.C. Wu, C.M. Truong, and D.W. Goodman, Phys. Rev. B 46, 12688 (1992).

${ }^{10}$ C. Sousa, G. Pacchioni, and F. Illas, Surf. Sci. 429, 217 (1999).

${ }^{11}$ A.M. Ferrari and G. Pacchioni, J. Phys. Chem. 99, 17010 (1995).

${ }^{12}$ F. Illas and G. Pacchioni, J. Chem. Phys. 108, 7835 (1998).

${ }^{13}$ F. Finocchi, J. Goniakowski, and C. Noguera, Phys. Rev. B 59, 5178 (1999).

${ }^{14}$ L. Ojamäe and C. Pisani, J. Chem. Phys. 109, 10984 (1998).
${ }^{15}$ G. Pacchioni and P. Pescarmona, Surf. Sci. 412/413, 657 (1998).

${ }^{16}$ J. Kramer, W. Ernst, C. Tegenkamp, and H. Pfnür, Surf. Sci. 517, 87 (2002).

${ }^{17}$ D. Peterka, C. Tegenkamp, K.-M. Schröder, W. Ernst, and H. Pfnür, Surf. Sci. 431, 146 (1999).

${ }^{18}$ P.R. Underhill and T.E. Gallon, Solid State Commun. 43, 9 (1982).

${ }^{19}$ P.A. Cox and A.A. Williams, Surf. Sci. Lett. 175, L782 (1986).

${ }^{20}$ C. Tegenkamp, Niedrigdimensionale Defektstrukturen in der Isolatorepitaxie Dissertation, Universität Hannover, Germany, 2000.

${ }^{21}$ Y. Chen, R.T. Williams, and W.A. Sibley, Phys. Rev. 182, 960 (1969).

${ }^{22}$ Y. Chen, J.L. Kolopus, and W.A. Sibley, Phys. Rev. 186, 865 (1969).

${ }^{23}$ H. Ibach and D. L. Mills, in Electron Energy Loss Spectroscopy and Surface Vibrations (Academic Press, New York, 1982).

${ }^{24}$ M.L. Knotek and P.J. Feibelman, Phys. Rev. Lett. 40, 964 (1978).

${ }^{25}$ M.L. Knotek and P.J. Feibelman, Surf. Sci. 90, 78 (1979).

${ }^{26}$ J.H. Lunsford and J.P. Jayne, J. Chem. Phys. 44, 1487 (1966).

${ }^{27}$ A. J. Tench and P. Holroyd, Chem. Commun. (London) 1968, 471 (1968).

${ }^{28}$ G. Pacchioni, A.M. Ferrari, and E. Giamell, Chem. Phys. Lett. 255, 58 (1996).

${ }^{29}$ A.M. Ferrari and G. Pacchioni, J. Chem. Phys. 107, 2066 (1997). 